\title{
An Elasticity Approach to the Newsvendor with Price-Sensitive Demand
}

\author{
Ayşe Kocabıyıkoğlu \\ Department of Management, Bilkent University, Bilkent, Ankara 06800, Turkey, aysekoca@ bilkent.edu.tr \\ loana Popescu \\ Decision Sciences Area, INSEAD, Singapore 138676, Republic of Singapore, ioana.popescu@insead.edu
}

\begin{abstract}
We introduce a measure of elasticity of stochastic demand, called the elasticity of the lost-sales rate, which offers a unifying perspective on the well-known newsvendor with pricing problem. This new concept provides a framework to characterize structural results for coordinated and uncoordinated pricing and inventory strategies. Concavity and submodularity of the profit function, as well as sensitivity properties of the optimal inventory and price policies, are characterized by monotonicity conditions, or bounds, on the elasticity of the lost-sales rate. These elasticity conditions are satisfied by most relevant demand models in the marketing and operations literature. Our results unify and complement previous work on price-setting newsvendor models and provide a new tool for researchers modeling stochastic price-sensitive demand in other contexts.
\end{abstract}

Subject classifications: inventory/production: newsvendor problem with pricing, coordination; marketing: pricing; economics: monopoly under uncertainty.

Area of review: Revenue Management.

History: Received September 2007; revisions received February 2009, November 2009; accepted April 2010.

\section{Introduction}

The importance and difficulty of understanding the interaction of pricing and inventory decisions in uncertain demand environments is well established and has motivated a vast literature on stochastic price-inventory problems (see, e.g., Tayur et al. 1999 and Chan et al. 2004 for surveys). The backbone of these operational models is the classical newsvendor with pricing model, which incorporates price sensitivity in the classical inventory problem with stochastic demand.

The existing newsvendor with pricing (NVP) literature is extensive, but has mainly focused on additive and multiplicative demand models, providing model-specific approaches and results. Our goal in this paper is to develop a unified approach and provide general, nonparametric conditions on demand for the NVP problem with lost sales to be well behaved in several respects.

We model demand as a general stochastic function of price, which encompasses additive-multiplicative models typically used in the NVP literature (Petruzzi and Dada 1999) as well as other relevant market-response models not covered by existing results. The latter notably include attraction models (e.g., logit or power), which are "among the most commonly used market share models, in both empirical studies and theoretical models" (Bernstein and Federgruen 2004, p. 874).

We propose a new measure, the lost-sales rate $(L S R)$ elasticity, for a given price and inventory level, which captures key structural properties of the NVP model under general, stochastic price-dependent demand. Based on this measure, we provide "nested" sets of conditions (from necessary and sufficient, to easy-to-verify sufficient ones) for the NVP objective and policy to satisfy desirable regularity and sensitivity properties under both coordinated and sequential decision processes.

In a perfectly coordinated environment, where price and quantity decisions are made simultaneously, we show that if LSR elasticity exceeds $1 / 2$ (globally, respectively, pathwise), the joint pricing and newsvendor problem is concave (jointly, respectively, pathwise), hence easy to solve, and admits a unique solution. Uniqueness is also guaranteed by increasing LSR elasticity.

In an uncoordinated setting, we investigate the optimal responses of marketing and operations divisions to a change in production and price, respectively. Necessary and sufficient conditions for price and inventory policies to be monotone are characterized by pathwise lower bounds of 1 on the LSR elasticity, along the corresponding optimal decision path. A global bound of 1 is equivalent to submodularity of expected profits. Monotonicity of the optimal price (respectively, inventory) policy is also ensured by LSR elasticity increasing in quantity (respectively, price).

In sum, we find that increasing LSR elasticity is an important property that guarantees uniqueness of the optimal coordinated NVP solution as well as sensitivity results for uncoordinated policies. We further argue that this property is not very restrictive. 
It is natural to assume that a stochastic demand function $\mathbf{D}(p)$ is decreasing in $p$ in some stochastic sense. It turns out that increasing LSR elasticity (with respect to quantity) is precisely equivalent to $\mathbf{D}(p)$ being stochastically decreasing in price in the hazard rate order (formally defined in §5), a stronger order than first-order stochastic dominance. We identify a general class of stochastic demand models with increasing LSR elasticity and show that it includes the majority of models studied in the NVP literature as well as other relevant market-response models, including attraction models. We further characterize what general demand properties drive the effect of uncertainty on optimal prices, extending existing comparisons with established riskless benchmarks. Thus, our results unify, generalize, and complement the existing NVP literature.

Literature. The vast literature on coordinated pricing and inventory decisions has been reviewed by Yano and Gilbert (2003) and Chan et al. (2004), and specifically for the newsvendor problem by Petruzzi and Dada (1999). Some representative NVP works include Karlin and Carr (1962), Lau and Lau (1988), Mills (1959, 1962), Nevins (1966), Yao et al. (2006), Young (1978), and Zabel (1970). These papers, and most of the literature, provide results for additive, multiplicative, or additive-multiplicative demand models. An exception is Raz and Porteus (2006), who use a general demand model (not comparable to ours) specified up to a finite number of empirically estimated fractiles, assumed piecewise linear in price.

Our model falls in the class of static NVP decision models with stochastic demand and lost sales. Both in terms of model and results, our paper is closest to Zabel (1970), Young (1978), Petruzzi and Dada (1999), Yao et al. (2006) and references within (cf. their Tables 1 and 2). Our results based on LSR elasticity are more general, and not dominated by any of these, as discussed in $\S 5$.

Our LSR-elasticity based conditions for the NVP model extend to richer inventory models, including multiproduct, flexible manufacturing and revenue management models (see Kocabiyikoglu et al. 2009), as well as some dynamic settings (see de Vericourt and Lobo 2009). In that respect, our work is potentially relevant to (albeit not directly comparable with) several streams of NVP literature, including multiproduct (e.g., Netessine and Rudi 2003), competitive (e.g., Zhao and Atkins 2008, Bernstein and Federgruen 2005), contracting (e.g., Ha 2001, Wang et al. 2004), and multiperiod models (e.g., Zabel 1972, Monahan et al. 2004, Netessine 2006). NVP models, where all demand is served at the set price and excess demand is backlogged at a priceindependent cost, preclude lost sales as a special casehence are not comparable to ours (e.g., Federgruen and Heching 1999, Agrawal and Seshadri 2000, and Chen and Simchi-Levi 2003).

Finally, our contributions are similar in spirit to Ziya et al. (2004) and Lariviere (2006), although the setup and results are not comparable. These authors discuss and unify important demand assumptions used for pricing and/or inventory problems. Their conditions translate desirable concavity properties of a deterministic revenue function into failure-rate conditions on the stochastic willingnessto-pay distribution underlying a deterministic price-demand function.

Structure. The rest of the paper is organized as follows. The model and underlying assumptions are presented in $\S 2$. Sections 3 and 4 translate properties of the optimal price and inventory policies in uncoordinated (respectively, coordinated) settings into bounds and monotonicity conditions on LSR elasticity. Conditions for monotone LSR elasticity are presented in $\S 5$, together with specific examples. The effect of uncertainty on optimal prices, as well as comparison with established riskless price benchmarks are studied in $\$ 6$. Finally, $\$ 7$ concludes the paper.

\section{The Model}

This paper considers a profit-maximizing firm seeking to optimize inventory $x$ and/or price $p$ decisions for a single product. These decisions are made either sequentially or simultaneously before observing an uncertain, pricedependent demand, $\mathbf{D}(p)$, and excess demand is lost. For simplicity, we assume a constant unit $\operatorname{cost} c$; all our results extend without loss of generality to increasing and convex cost functions $c(x)$. In a coordinated setting (studied in $\S 4$ ), the firm jointly determines price and quantity decisions, $\left(p^{* *}, x^{* *}\right)$, which maximize expected profit:

$$
\begin{aligned}
& \max _{p, x} \Pi(p, x), \quad \text { where } \\
& \qquad \Pi(p, x)=p \mathbb{E}[\min (\mathbf{D}(p), x)]-c x .
\end{aligned}
$$

The constrained (or truncated) revenue is denoted by

$R(p, x)=p \mathbb{E}[\min (\mathbf{D}(p), x)]$.

In an uncoordinated environment (studied in $\S 3$ ), a sales and marketing division (or a price-setting firm) sets prices $p^{*}(x)$ by optimizing the objective $\Pi(p, x)$ in $(1)$ for a given inventory $x$, whereas an operations division (or quantitysetting firm) sets inventory levels $x^{*}(p)$ by optimizing $\Pi(p, x)$ with respect to $x$, for a given $p$.

\subsection{Demand Model}

The price-dependent stochastic demand is modeled as

$\mathbf{D}(p)=d(p, \mathbf{Z})$,

where $d$ is a deterministic demand function, and $\mathbf{Z}$ is a random variable with price-independent cdf $\Phi$ and density $\phi$, capturing demand risk. In empirical estimation, $\mathbf{Z}$ can be random noise, or an independent variable in a (possibly nonlinear) regression model. Conceptually, it is convenient to think of $\mathbf{Z}$ as a sales driver that is uncertain, or not 
perfectly controlled by the decision maker, or the relevant division of the firm. Examples include market size, personal disposable income, product quality, advertising spend, or a reference price (see, e.g., Hanssens et al. 2001).

The demand function $d(p, z)$ is decreasing in price $p$, strictly increasing in $z$, and twice differentiable in $p$ and $z$. Throughout the paper the terms increasing/decreasing, positive/negative are used in their weak sense. Monotonicity of $d$ allows us to uniquely define the stocking factor $z(p, x)$ as the value of the sales driver $z$ for which demand perfectly matches supply, $d(p, z(p, x))=x$. (This is consistent with the model-specific stocking factor definitions in Petruzzi and Dada 1999, but their normalized mean-variance interpretation is limited to additive and multiplicative settings.) The riskless (or pathwise) unconstrained revenue is denoted $r(p, z)=p d(p, z)$; we assume $r(p, z)$ is strictly concave in $p$ for any risk realization $z$. Denoting partial derivatives by corresponding subscripts, this condition amounts to $2 d_{p}(p, z)+p d_{p p}(p, z)<0$.

The general demand model (3) encompasses the additive and multiplicative demand models commonly used in the NVP literature, as well as additive-multiplicative models (Young 1978):

$\mathbf{D}(p)=d(p, \mathbf{Z})=\alpha(p) \mathbf{Z}+\beta(p)$,

where $\alpha(p), \beta(p)$ are decreasing functions of $p$. Setting $\alpha(p) \equiv 1$ gives the additive model (price influences the location of the demand distribution), whereas $\beta(p) \equiv 0$ gives the multiplicative model (price influences demand scale).

Additive-multiplicative models, although easy to estimate, make restrictive implicit assumptions that drive the nature of NVP results (see, e.g., §6). For example, such models imply a monotone relationship between price and demand variability, as measured by variance and coefficient of variation (see Petruzzi and Dada 1999, p. 187). Empirical observations, however, suggest that these relationships may not hold, in which case existing parametric approximations for the NVP problem perform poorly (see Raz and Porteus 2006, p. 1765). Our general demand model fills this gap by making no a priori assumptions regarding the effect of price on demand variability. Moreover, model (3) encompasses several practically relevant marketresponse models, used in the marketing, economics, and operations literature, that are not additive-multiplicative, including attraction models such as logit and power choice models, further described in $\$ 5.2$.

\subsection{Lost-Sales Rate (LSR) Elasticity}

For a given price $p$ and stock $x$, the lost-sales rate $(L S R)$ is denoted $q(p, x)=1-F(p, x)$, where $F(p, x)=$ $P(\mathbf{D}(p) \leqslant x)$ is the demand cdf, or probability of no lost sale. The keystone for our developments is a new elasticity concept, the price elasticity of the rate of lost sales $q$, for a given price $p$ and quantity level $x$. Specifically, the LSR elasticity is the percentage change in the rate of lost sales with respect to the percentage change in price for a given quantity.

DEFINITION 1. The LSR elasticity for a given price $p$ and inventory level $x$ is defined as

$\mathscr{E}(p, x)=-\frac{p q_{p}(p, x)}{q(p, x)}=\frac{p F_{p}(p, x)}{1-F(p, x)}$.

The LSR elasticity $\mathscr{E}(p, x)$ combines the relative sensitivity of (lost) sales with respect to its underlying factors, inventory, and price. Our results in this paper characterize relevant structural properties of the NVP problem in terms of (necessary and) sufficient conditions on $\mathscr{E}$, suggesting that this particular elasticity concept captures essential features of the NVP setup. A more detailed discussion on the LSR elasticity is provided in $\S 5$.

Throughout the paper, price and quantity are implicitly optimized over (positive) compact intervals $p \in P=$ $\left[p_{L}, p_{H}\right], x \in X=\left[x_{L}, x_{H}\right]$, where $p_{H}, x_{H}$ are arbitrary, possibly infinite, and $p_{L} \geqslant c$. We set $x_{L}=0$ without loss of generality, and $p_{L}=\arg \max \left\{d\left(p, \Phi^{-1}(1-c / p)\right)\right.$; $p \geqslant c\}$; our results hold for any subintervals of $P$ and $X$ thus defined. The lower bound on price, $p_{L}$, helps to express price monotonicity of the optimal inventory level in terms of LSR elasticity (in Theorem 1b). This bound is in a sense necessary, as argued in $\S 3$, and not very restrictive. For example, for additive models $a-b p+\mathbf{Z}$, with $\mathbf{Z}$ uniform on $[0,1]$ or exponential with mean 1 , we obtain $p_{L}=c$ whenever $b \geqslant 1 / c$, effectively imposing no additional restriction on the admissible price range.

A glossary of notation is provided in the appendix.

\section{Price-Inventory Interactions in a Sequential Decision Process}

In most practical settings, price and inventory decisions are not managed simultaneously, but rather sequentially, by separate units of the firm (Zhao and Atkins 2008); sales and marketing divisions set prices based on planned inventory, whereas operations (or supply chain planning) divisions decide production or inventory levels based on preset prices.

Economic considerations suggest that lower prices lead to higher mean demand, and consequently should drive up the preferred inventory levels. On the other hand, stochastic inventory theory predicts that lower prices lead to lower underage costs, and hence lower safety stocks, driving inventory levels down. These two arguments suggest that under demand uncertainty the relationship between price and quantity is generally ambiguous, as previously acknowledged in the literature (Zabel 1970, Raz and Porteus 2006). This section presents conditions, in terms of LSR elasticity, for optimal price and inventory policies, $p^{*}(x)$ and $x^{*}(p)$, respectively, to be monotone in such uncoordinated settings. These policies are obtained by optimizing $\Pi(p, x)$ in (1) with respect to $p$ and $x$, respectively, 
with the other variable as a parameter (see Lemma 1 in the appendix).

A widely used sufficient condition for comparative statics is submodularity of the objective function. (A differentiable function $\mathfrak{f}(x, y)$ is submodular if it has a negative cross derivative $\tilde{f}_{x y}(x, y) \leqslant 0$ supermodularity is defined by the opposite inequality.) All proofs are provided in the appendix.

Proposition 1. $\Pi(p, x)$ is submodular if and only if $\mathscr{E}(p, x) \geqslant 1$ for all $p$ and $x$. In this case, the inventory and pricing policies $x^{*}(p)$ and $p^{*}(x)$ are decreasing in their respective arguments.

Characterizing submodularity of the NVP objective can be useful to obtain structural properties in more complex (e.g., dynamic or competitive) settings. De Vericourt and Lobo (2009) use the elasticity bound of 1 to obtain price monotonicity (and pathwise concavity) in a dynamic model of nonprofit operations; these authors emphasize the importance of obtaining stronger sufficient conditions that can be propagated in a dynamic setting. In general, checking $\mathscr{E}(p, x) \geqslant 1$ amounts to bounds on $p$ and/or $x$. For example, for $\mathbf{D}(p)=a-b p+\mathbf{Z}, \mathbf{Z} \sim$ Exponential $(1), \mathscr{E}(p, x) \geqslant 1$ as long as $p>1 / b$.

The uniform lower bound of 1 on $\mathscr{E}$ is sufficient, but not necessary for the optimal policies to be monotone. Necessary and sufficient conditions are characterized by a lower bound of 1 on the LSR elasticity along the optimal decision paths, denoted $\mathscr{E}^{*}(p)=\mathscr{E}\left(p, x^{*}(p)\right)$ and $\mathscr{E}^{*}(x)=$ $\mathscr{E}\left(p^{*}(x), x\right)$. Alternative sufficient conditions, based on monotonicity of $\mathscr{E}(p, x)$ in $p$ or $x$, are potentially easier to verify because they do not require evaluation of $\mathscr{E}$ along an optimal policy path.

Theroem 1. (a) The optimal pricing policy $p^{*}(x)$ is decreasing in $x$ if and only if $\mathscr{E}^{*}(x) \geqslant 1$ for all $x$. In particular, this holds if $\mathscr{E}(p, x)$ is increasing in $x$.

(b) The optimal inventory policy $x^{*}(p)$ is decreasing in $p$ if and only if $\mathscr{E}^{*}(p) \geqslant 1$ for all $p$. In particular, this holds if $\mathscr{E}(p, x)$ is increasing in $p$.

Sufficiency of the increasing LSR elasticity condition in Theorem 1(b) relies on the lower bound $p_{L}$ on price, defined in $\$ 2.2$. Lemma 3 in the appendix indicates that this bound is actually necessary, i.e., $x^{*}(p)$ decreasing implies $p \geqslant p_{L}$. Similar lower bounds on price, which ensure that demand elasticity exceeds 1 , have been used, for example, by Ziya et al. (2004, 2006).

\section{Simultaneous Price-Inventory Optimization}

This section focuses on jointly optimized price and quantity decisions, corresponding to a setting where these decisions are centrally managed by the headquarters of the firm and/or marketing and operations divisions are fully coordinated ( $\mathrm{Li}$ and Atkins 2005). We present various sets of alternative conditions for the objective in (1) to have a unique solution $\left(p^{* *}, x^{* *}\right)$; this problem is referred to as the newsvendor with pricing (NVP) problem.

Proposition 2. If $\mathscr{E}(p, x) \geqslant 1 / 2$, then $\Pi(p, x)$ is jointly concave in $p$ and $x$, and the (NVP) problem has a unique price-quantity solution.

Note that the deterministic revenue function $p \min (d(p$, $z(p, x)), x)$ is not jointly concave in $(p, x)$, even when $r(p, z)=p d(p, z)$ is concave. Our result suggests that with sufficient variability in excess demand guaranteed by the elasticity bound, the extreme effect of the deterministic case can be smoothed out. A similar effect was observed numerically by Netessine and Rudi (2003) for a multiproduct newsvendor model. Similar bounds on different elasticity measures have been used in the literature to ensure regularity, e.g., by Bernstein and Federgruen (2005), Ziya et al. (2006), and de Vericourt and Lobo (2009).

THEROEM 2. The following alternative conditions are sufficient for the (NVP) problem to have a unique optimal price-quantity solution:

(a) $\mathscr{E} *(x) \geqslant 1 / 2$ for all $x$; in this case, $\Pi^{*}(x)=$ $\Pi\left(p^{*}(x), x\right)$ is concave in $x$.

(b) $\mathscr{E}^{*}(p) \geqslant 1 / 2$ or all $p$; in this case, $\Pi^{*}(p)=$ $\Pi\left(p, x^{*}(p)\right)$ is concave in $p$.

(c) $\mathscr{E}(p, x)$ is increasing in $x$ or in $p$.

This result allows us to solve the NVP problem numerically as a one-dimensional concave optimization problem and guarantees uniqueness of the optimal solution $\left(p^{* *}, x^{* *}\right)$. We further argue that in some cases, the (global and pathwise) $1 / 2$ lower bounds are not only sufficient but also necessary for the corresponding regularity conditions. Therefore, no weaker constant bound can be expected to hold for all demand functions. Indeed, the bounds are tight for additive linear models, widely studied in the literature (e.g., Mills 1959, Lau and Lau 1988, Ha 2001, Petruzzi and Dada 1999, Zhao and Atkins 2008).

REMARK 1. If $d$ is linear in $p$, i.e., $d(p, z)=\delta(z) p+\gamma(z)$, then (a) $\mathscr{E} \geqslant 1 / 2$ is necessary and sufficient for joint concavity of $\Pi(p, x)$, and (b) $\mathscr{E}^{*}(\cdot) \geqslant 1 / 2$ is necessary and sufficient for concavity of $\Pi^{*}(\cdot)$.

Our results in this section fully generalize those of Zabel (1970), Young (1978), and Yao et al. (2006), all of which have increasing LSR elasticity, as argued in $\$ 5$. In terms of approach, the majority of the NVP literature and beyond (e.g., Zabel 1970, Young 1978, Wang et al. 2004, de Vericourt and Lobo 2009) obtains the optimal priceinventory policy by optimizing the NVP problem along the optimal price path. Only Whitin (1955), for deterministic demand, and Yao et al. (2006), use the opposite sequence, optimizing the pricing problem $\Pi^{*}(p)$ along the optimal quantity path $x^{*}(p)$. Their approaches rely on the additivemultiplicative setup and specific assumptions on $\mathbf{Z}$ (e.g., uniform/exponential in Zabel 1970, logconcave densities in 
Table 1. LSR elasticity conditions for the NVP solution.

\begin{tabular}{|c|c|c|}
\hline Unique $\left(p^{* *}, x^{* *}\right)$ & $p^{*}(x) \downarrow x$ & $x^{*}(p) \downarrow p$ \\
\hline $\begin{array}{l}\mathscr{E}(p, x) \uparrow p \text { or } x \\
\mathscr{E}(p, x) \geqslant 1 / 2\end{array}$ & $\begin{array}{l}\mathscr{E}(p, x) \uparrow x \\
\mathscr{E}(p, x) \geqslant 1\end{array}$ & $\begin{array}{l}\mathscr{E}(p, x) \uparrow p \\
\mathscr{E}(p, x) \geqslant 1\end{array}$ \\
\hline $\mathscr{E}^{*}(x)$ or $\mathscr{E}^{*}(p) \geqslant 1 / 2$ & $\mathscr{E} *(x) \geqslant 1\left(\frac{\ddagger}{*}\right)$ & $\mathscr{E} *(p) \geqslant 1\left(\frac{\vec{t}}{)}\right)$ \\
\hline
\end{tabular}

$\left.{ }^{\ddagger}\right)$ These conditions are necessary and sufficient.

Young 1978), whereas our analysis is general, resting on the general properties of the LSR elasticity.

Table 1 provides a summary of the alternative LSR elasticity conditions presented in $\S \S 3$ and 4 , which guarantee relevant properties of the NVP objective function and optimal price and inventory policies in coordinated and sequential environments. ${ }^{1}$ Together, these results suggest that the LSR elasticity concept introduced in this paper captures essential features of the newsvendor setup. In $\$ 5$ we show how the conditions provided in Table 1 can be verified in terms of properties of the riskless demand $d$ and the risk distribution $\mathbf{Z}$, and characterize a general class of demand models with increasing LSR elasticity.

\section{Monotone LSR Elasticity}

This section presents conditions for monotonicity of $\mathscr{E}(p, x)$ in quantity $x$ and price $p$, respectively, which are the key drivers of concavity and sensitivity results obtained in $\S \S 3$ and 4, as well as examples of relevant demand forms satisfying these properties.

\subsection{Demand Conditions for Increasing LSR Elasticity}

It is natural to assume that a stochastic demand function $\mathbf{D}(p)$ is decreasing in $p$, in some stochastic sense. It turns out that $\mathscr{E}(p, x)$ increasing in $x$ is precisely equivalent to $\mathbf{D}(p)$ being stochastically decreasing in price in the hazard rate order, ${ }^{2}$ or equivalently, the demand failure (or hazard) rate, $h_{D}(p, x)=f(p, x) / q(p, x)$, being increasing in price.

Proposition 3. $\mathscr{E}(p, x)$ is increasing in $x$ if and only if $\mathbf{D}(p)$ is decreasing in $p$ in the hazard rate order. If, moreover, the riskless price-elasticity $\epsilon_{P}(p, x)=\left(-p d_{p}\right.$ $(p, z(p, x))) /(d(p, z(p, x)))$ is increasing in $p$, then $\mathscr{E}(p, x)$ is also increasing in $p$.

We next provide a collection of relatively general sufficient conditions separating the riskless demand $d$ and the distribution $\mathbf{Z}$. By definition, $\mathbf{Z}$ is IFR (respectively, IGFR) if it has increasing failure rate, $h_{Z}(z)=\phi(z) /(1-\Phi(z))$, (respectively, generalized failure rate, $g_{Z}(z)=z h_{Z}(z)$ ).

Proposition 4. (a) Suppose that $\mathbf{Z}$ is IFR. If $d_{p} / d_{z}$ is decreasing in $z$, then $\mathscr{E}(p, x)$ is increasing in $x$. If, moreover, $p d_{p} / d_{z}$ is decreasing in $p$, then $\mathscr{E}(p, x)$ increases in $p$. (b) Suppose $\mathbf{Z}$ is IGFR. If the riskless cross elasticity $\tilde{\epsilon}_{P Z}(p, z)=\left(-p d_{p}(p, z)\right) /\left(z d_{z}(p, z)\right)$ is increasing in $z$, then $\mathscr{E}(p, x)$ is increasing in $x$. If, moreover, $\tilde{\epsilon}_{P Z}(p, z)$ increases in $p$, then $\mathscr{E}(p, x)$ increases in $p$.

The IFR and IGFR assumptions on $\mathbf{Z}$ are commonly used in the NVP literature and impose "very mild restrictions on the demand distribution" (Wang et al. 2004, p. 37). The weaker IGFR assumption "captures most common distributions a modeler would choose to employ" (Tayur et al. 1999, p. 241; also Lariviere and Porteus 2001). IFR distributions are those with log-concave survival functions and include distributions with log-concave density (also known as PF2); examples include uniform, exponential, normal, truncated normal, and lognormal distributions (see Barlow and Proschan 1996, and Bagnoli and Bergstrom 2005). All IFR distributions are IGFR, but the reverse is not true; for example, Gamma and Weibull distributions are IFR over a restricted set of parameters, but IGFR for all. For more on IFR and IGFR distributions, see Barlow and Proschan (1996), Lariviere (2006), or Lariviere and Porteus (2001).

We argue that the generic conditions on $d$ and $\mathbf{Z}$ in Proposition 4 are, relative to each other, as general as possible. They are "conditionally necessary" for $\mathscr{E}$ to be monotone in $x$ in the following weak sense: for an arbitrary, given function $d(p, z)$, if the corresponding LSR elasticity is increasing in $x$, for all IFR distributions $\mathbf{Z}$, then one can show that $d_{p} / d_{z}$ must be decreasing in $z$. Conversely, for an arbitrary, given $\mathbf{Z}$, if $\mathscr{E}(p, x)$ corresponding to $d(p, \mathbf{Z})$ is increasing in $x$, for all demand functions $d$ satisfying the given conditions, then $\mathbf{Z}$ must be IFR. Similar results hold for part (b) of the proposition.

The following simpler (but stronger) sufficient conditions are derived from Proposition 4(a).

Corollary 1. Suppose that $\mathbf{Z}$ is IFR and $d_{z z} \leqslant 0$ and $d_{p z} \leqslant 0$. Then $\mathscr{E}(p, x)$ is increasing in $x$. If, moreover, the riskless price elasticity, $\epsilon_{P}(p, x)=\left(-p d_{p}(p, z(p, x))\right) /$ $(d(p, z(p, x)))$, is increasing in $p$, in particular if $d_{p}+$ $p d_{p p} \leqslant 0$, then $\mathscr{E}(p, x)$ increases in $p$.

Diminishing demand sensitivity in response to a sales driver, $d_{z z} \leqslant 0$, is a common, empirically validated assumption (see, e.g., Hanssens et al. 2001, p. 95). Submodularity of riskless demand $\left(d_{p z} \leqslant 0\right)$ implies that a change in the uncontrollable sales driver has a greater impact on demand at lower price levels (and vice versa); our results in $\$ 6$ suggest that $d_{p z} \leqslant 0$ is necessary for the NVP problem to be well behaved in other important respects. Both conditions hold for additive-multiplicative models. Finally, the increasing (riskless) price-elasticity property (IPE) is commonly used in the NVP literature, e.g., by Ziya et al. (2006), Netessine (2006), and Yao et al. (2006), and holds, for example, if $d$ is concave in $p$; in particular, $d_{p}+p d_{p p} \leqslant 0$ implies concavity of the riskless revenue $r$.

The NVP literature so far has focused almost exclusively on additive and multiplicative demand models. The next 
result gives necessary and sufficient conditions for the LSR elasticity to be increasing in $x$ in such models, as well as sufficient conditions for monotonicity in $p$.

Corollary 2. Consider the additive-multiplicative model $d(p, z)=\alpha(p) z+\beta(p)$, as in (4).

(a) If $\mathscr{E}(p, x)$ is increasing in $x$, then $\mathbf{Z}$ is IGFR. If $\mathbf{Z}$ is IFR, then $\mathscr{E}(p, x)$ is increasing in $x$; if, moreover, $p \alpha^{\prime}(p)$ and $p \beta^{\prime}(p)$ are decreasing in $p$, then $\mathscr{E}(p, x)$ is also increasing in $p$.

(b) For multiplicative models $(\beta(p)=0), \mathscr{E}(p, x)$ is increasing in $x$ if and only if $\mathbf{Z}$ is IGFR. If, moreover, $\alpha(p)$ has increasing elasticity, then $\mathscr{E}(p, x)$ is also increasing in $p$.

(c) For additive models $(\alpha(p)=1), \mathscr{E}(p, x)$ is increasing in $x$ if and only if $\mathbf{Z}$ is IFR. If, moreover, $p \beta^{\prime}(p)$ is decreasing in $p$, then $\mathscr{E}(p, x)$ is also increasing in $p$.

This corollary encompasses most existing NVP results. Yao et al. (2006) obtain uniqueness of the optimal coordinated solution for general multiplicative (respectively, additive) models assuming that $\mathbf{Z}$ is IGFR (respectively, IFR) and price elasticity of $\alpha(p)$ (respectively, $\beta(p)$ ) is increasing. Under these assumptions, Corollary 2 ensures that $\mathscr{E}(p, x)$ is monotone in both $p$ and $x$. Their setup already encompasses most of the existing models in the literature, as summarized in their Tables 1 and 2. Additive models usually assume a linear price dependence and IFR (in particular uniform) risk (e.g., Mills 1959, Lau and Lau 1988, Ha 2001, Zhao and Atkins 2008). Multiplicative models are either isoelastic with IGFR risk (Wang et al. 2004, Monahan et al. 2004), or general with exponential or uniform risk, hence IFR (Zabel 1970). All of these, as well as the additive-multiplicative model of Young (1978, with logconcave or lognormal risk) satisfy monotone LSR elasticity, in light of Corollary 2. Petruzzi and Dada (1999) study additive linear and multiplicative isoelastic demand models. Our results show that IGFR of $\mathbf{Z}$ ensures regularity in both models. However, they assume that $\mathbf{Z}$ satisfies $2 h_{Z}^{2}+h_{Z}^{\prime} \geqslant 0$, which holds for all IFR distributions but is not comparable to IGFR (see Ziya et al. 2004, Conditions 1 and 3). Therefore, Petruzzi and Dada's (1999) regularity conditions are generally not comparable to our monotone LSR elasticity conditions.

\subsection{Examples of Demand Models with Monotone LSR Elasticity}

For illustration purposes, we consider some relevant demand models and characterize conditions on $\mathbf{Z}$ for these to have increasing LSR elasticity. We find that increasing LSR elasticity is usually equivalent to IFR or IGFR of $\mathbf{Z}$.

Attraction models, such as the logit $a\left(e^{z-b p}\right) /\left(1+e^{z-b p}\right)$ and power $a\left(z /\left(z+p^{b}\right)\right)$ models (e.g., Phillips 2005, Agrawal and Ferguson 2007), are among the most commonly used market share models, both in empirical studies and theoretical models, and emerge naturally from intuitive axioms (Bernstein and Federgruen 2004, pp. 873-874).
These models assume a fixed market size, $a$, from which the firm acquires a market share that is proportional to (an attraction value given by) a function of its price ( $b$ measures price sensitivity), and possibly other sales drivers, captured by $z$ (Hanssens et al. 2001). These models are not amenable to additive-multiplicative forms, so, albeit widely used in both theory and practice, they are not covered by previous NVP results.

A ubiquitous model in the economic and operations literature is the heterogeneous willingness-to-pay model, $d(p, z)=z P(\mathbf{W} \geqslant p)$ (see, e.g., Phillips 2005), where $\mathbf{W}$ denotes consumers' willingness-to-pay distribution, and $z$ is the market size. Unlike attraction models, where market size is fixed, this model assumes that demand uncertainty comes from market size (a common assumption, e.g., for learning models), leading to a multiplicative demand model. IGFR of the willingness-to-pay distribution is a common assumption in the literature, ensuring regularity of the deterministic objective when market size is known (e.g., Lariviere 2006, Ziya et al. 2004).

Finally, Table 2 also presents conditions for additive linear and multiplicative isoelastic models, commonly used in the NVP literature (Petruzzi and Dada 1999), as well as exponential and log demand models (see Hanssens et al. 2001). The exponential model, $d(p, z)=e^{z-b p}$, can be transformed into a multiplicative model by a change of variables $v=e^{z}$; the IFR condition on $\mathbf{Z}$ is equivalent to IGFR of $e^{\mathbf{Z}}$. The $\log$-model is not amenable to additivemultiplicative form.

\section{The Effect of Uncertainty and Riskless Benchmarks}

We conclude by addressing one of the issues commonly discussed in the NVP literature: the relationship between the optimal NVP price and a riskless price benchmark (see Petruzzi and Dada 1999). We also characterize the effect of changes in uncertainty on optimal prices, under our general demand model. Our results suggest that properties of riskless demand and revenue/profit drive these results and explain the effect of uncertainty on optimal prices.

Two price benchmarks are commonly used in the literature:

- The riskless price: $p^{0}=\arg \max (p-c) \mathbb{E}[\mathbf{D}(p)] \max$ imizes profit for average demand.

- The base price: $p_{B}(z)=\arg \max _{p}(p-c) \mathbb{E}[d(p$, $\min (\mathbf{Z}, z))]=\mathbb{E}[\pi(p, \min (\mathbf{Z}, z))]$ maximizes profit from expected sales for a given stocking factor $z$; here $\pi(p, z)=$ $(p-c) d(p, z)$ denotes the riskless profit function. These extend Petruzzi and Dada's (1999) definitions in our general setting.

Define $p^{*}(z)=\arg \max \Pi(p, d(p, z))=\arg \max \mathbb{E}[r(p$, $\min (\mathbf{Z}, z))]-c d(p, z)$, the optimal price for a given stocking factor $z$.

Proposition 5. (a) If $d_{p z} \geqslant 0$, then $p^{*}(z) \leqslant p_{B}(z)$ for all $z$, and $p^{* *} \leqslant p^{0}$. 
Table 2. Necessary and sufficient conditions on $\mathbf{Z}$ for increasing LSR elasticity.

\begin{tabular}{lcccc}
\hline Demand model & $d(p, z)$ & $\mathscr{E}(p, x)$ & $\mathscr{E}(p, x) \uparrow x$ & $\mathscr{E}(p, x) \uparrow p$ \\
\hline additive linear & $z-b p$ & $b p h_{Z}$ & IFR & IGFR \\
multiplicative isoelastic & $a p^{-b} z$ & $b g_{Z}$ & IGFR & IGFR \\
power & $a \frac{z}{z+p^{: b}}$ & $b g_{Z}$ & IGFR & IGFR \\
& $a \frac{e^{z-b p}}{1+e^{z-b p}}$ & $b p h_{Z}$ & IFR & IGFR \\
logit & $e^{z-b p}$ & $b h_{Z}$ & IFR & IFR \\
exponential & $\log _{a}(z-b p)$ & $b p h_{Z}$ & IFR & IGFR $^{\dagger}$ \\
$\log$ & $z P(\mathbf{W} \geqslant p)$ & $g_{W}(p) g_{Z}$ & IGFR & ${\text { IGFR } \& \mathbf{W} \text { IGFR }^{\dagger}}^{\text {economic wtp }}$ \\
\hline
\end{tabular}

${ }^{\dagger}$ Conditions are only sufficient.

(b) If $d_{p z} \leqslant 0$, then $p^{*}(z) \geqslant p_{B}(z)$ for all $z$. If, moreover, $\pi_{p z} \leqslant 0$, or $d(p, z)=\alpha(p) z$, then $p^{* *} \geqslant p^{0}$.

The relationship between the optimal NVP price and the riskless price, as identified in the NVP literature, is mixed; Mills (1959) shows that $p^{* *} \geqslant p^{0}$ for additive models, whereas Karlin and Carr (1962) find the opposite for multiplicative models. Petruzzi and Dada (1999) introduce the base price $p_{B}^{*}(z)$ in order to resolve this inconsistency and show that it is a lower bound on the optimal price (for additive linear and multiplicative isoelastic models). Proposition 5(a) indicates that their result extends to general demand models as long as $d_{p z} \leqslant 0$. This condition, discussed in $\S 5$, holds for all additive-multiplicative demand models. Proposition 5(b) further suggests that the base price in Petruzzi and Dada (1999) is not a robust lower bound and can become an upper bound if $d_{p z} \geqslant 0$ (e.g., for $d(p, z)=\log (z-b p))$.

We recover Petruzzi and Dada's (1999) result for additive linear models $\left(p_{B}(z)=p^{*}(z), p^{0} \geqslant p^{* *}\right)$ and show that it extends for all additive models $\left(d_{p z}=0\right)$. We also recover their result for multiplicative isoelastic models $\left(p_{B}(z) \leqslant\right.$ $\left.p^{*}(z), p^{0} \leqslant p^{* *}\right)$ and show that it extends for all multiplicative models. It also extends for additive-multiplicative models with elastic riskless demand (i.e., elasticity of $\alpha(p)$ exceeding 1), and more generally, whenever $\pi_{p z} \leqslant 0$. (If $d_{p z} \leqslant 0$ and $\pi_{p z} \geqslant 0$, we can show that $p_{B}(z) \leqslant p^{0}$, but the relation between $p^{0}$ and $p^{* *}$ remains ambiguous.)

We further investigate the sensitivity of optimal prices to changes in uncertainty, as captured by first-order dominance and the convex order: $\mathbf{Z}_{L} \succeq_{F S D} \mathbf{Z}$, is stochastically larger than $\mathbf{Z}$ and $\mathbf{Z}_{V} \succeq_{C X} \mathbf{Z}$ is more variable than $\mathbf{Z}{ }^{3}$ Let $p_{i}^{*}(z)=\arg \max \mathbb{E}\left[r\left(p, \min \left(\mathbf{Z}_{i}, z\right)\right)\right]-c d(p, z), i=L, V$. These results enable the comparison with another riskless benchmark, $p^{0}(z)=\arg \max p d(p, \min (\mu, z))-c d(p, z)$, the price that maximizes profit when $\mathbf{Z}$ is replaced by its mean $\mu=\mathbb{E}[\mathbf{Z}]$.

Proposition 6. Suppose that $\mathbf{Z}_{L} \succeq_{F S D} \mathbf{Z}$, and $\mathbf{Z}_{V} \succeq_{C X} \mathbf{Z}$.

(a) If $r_{p z} \geqslant 0$, then $p_{L}^{*}(z) \geqslant p^{*}(z)$. If in addition $r_{p z z} \leqslant 0$, then $p_{V}^{*}(z) \leqslant p^{*}(z) \leqslant p^{0}(z)$. (b) If $r_{p z} \leqslant 0$, then $p_{L}^{*}(z) \leqslant p^{*}(z)$. If in addition $r_{p z z} \geqslant 0$, then $p_{V}^{*}(z) \geqslant p^{*}(z) \geqslant p^{0}(z)$.

We find that higher demand, triggered by a firstorder increase in the sales driver, leads to higher optimal prices if the riskless revenue $r$ is supermodular; the effect of variability, as captured by the convex order, also depends on this condition (and a third-order condition, which always holds for additive-multiplicative models $r_{p z z}=0$ ). Supermodularity of $r$ means that marginal revenue increases with $z$, i.e., $d_{z}$ is price inelastic. This holds whenever $d_{p z} \geqslant 0$, and in particular for all additive models $\left(r_{p z}=1\right)$. For multiplicative and additive-multiplicative models, $r_{p z} \geqslant 0$ whenever (riskless) demand is price inelastic (i.e., $\alpha(p)+p \alpha^{\prime}(p) \geqslant 0$ ). In particular, we find that $p^{*}(z) \leqslant p^{0}(z)$ for all additive models, and for additivemultiplicative models with inelastic $\alpha(p)$, whereas $p^{*}(z) \geqslant$ $p^{0}(z)$ if $\alpha(p)$ is price elastic.

In summary, the results in this section suggest that the relationship with riskless price benchmarks may not be driven by the effect of price on variance and coefficient of variation of demand, as speculated in the literature (Petruzzi and Dada 1999, pp. 186-187), but primarily by the properties of (riskless) demand and profit. In particular, for additive-multiplicative models the results depend on whether or not the multiplicative demand component is price elastic.

\section{Conclusion}

We introduce a new elasticity concept that provides a framework to study interdependent pricing and inventory decisions with stochastic demand. Focusing on the singleproduct price-setting newsvendor model, our key contribution is to characterize general models of stochastic price-dependent demand, which guarantee relevant structural properties of the optimal price and inventory policies. These include uniqueness and sensitivity properties of the joint price-inventory solution in a coordinated system, and of the optimal pricing and quantity policies in a sequential decision framework. Our approach relies on a new measure of demand elasticity, the elasticity of the lost-sales 
rate, or LSR elasticity, whose properties-in particular monotonicity-drive the desired results. We further characterize general classes of demand models with increasing LSR elasticity. These classes unify, generalize, and complement assumptions commonly made in the NVP literature, such as additive-multiplicative models and failure-rate conditions. We expect these results to be useful for modeling stochastic, price-dependent demand in other operational settings.

\section{Appendix. Proofs}

For convenience, we provide in Table 3 a summary of notation frequently used throughout the paper and appendix. We denote the evaluation of any function $\mathfrak{f}$ along the optimal policy path $x^{*}(p)$ as $\mathfrak{f}^{*}(p)=\left.\mathfrak{f}(p, x)\right|_{x=x^{*}(p)}=\mathfrak{f}\left(p, x^{*}(p)\right)$, for example, $\Pi^{*}(p)=\Pi\left(p, x^{*}(p)\right)$. Similarly, $\mathfrak{f}^{*}(x)=$ $\left.\mathfrak{f}(p, x)\right|_{p=p^{*}(x)}$. The generic argument of $\mathfrak{f}^{*}$ makes the evaluation path unambiguous. Denote $\mathfrak{f}_{x}^{*}(p)=\left.\mathfrak{f}_{x}(p, x)\right|_{x=x^{*}(p)}$ and $\mathfrak{f}_{x}^{*}(x)=\left.\mathfrak{f}_{x}(p, x)\right|_{p=p^{*}(x)}$, the corresponding derivatives evaluated at the optimal quantity and price, respectively. In this notation, the derivative always precedes functional evaluation.

Proof of Proposition 1. Taking the derivative of the marginal revenue, $R_{x}(p, x)=p q(p, x)$, with respect to $p$, we obtain

$R_{x p}=q(p, x)+p q_{p}(p, x)=q(p, x)(1-\mathscr{E}(p, x))$,

where the second equality is obtained using (5). It follows that $R_{x p} \leqslant 0$ whenever $\mathscr{E} \geqslant 1$. Monotonicity of $x^{*}(p)$ and $p^{*}(x)$ follows by Topkis' Theorem (see Topkis 1998, Theorem 2.8.2).
The proof of Theorem 1 relies on the following lemmas:

LEMma 1. (a) For any given price $p$, the optimal order quantity $x^{*}(p)$ is unique and solves $q(p, x)=c / p$.

(b) For any given quantity $x$, the optimal price $p^{*}(x)$ is unique and solves

$\int_{0}^{x} q(p, v)(1-\mathscr{E}(p, v)) d v=0$.

Proof. Because the minimum of concave functions is concave, $R(p, x)=\mathbb{E}[\min (r(p, \mathbf{Z}), p x)]$ is concave in $p$ (because $r(p, z)$ is concave in $p$ ) and in $x$; this implies uniqueness of $p^{*}(x)$ and $x^{*}(p)$. Part (a) states the wellknown critical fractile inventory solution. Equation (7) states the first-order condition with respect to $p$. To see this, write (2) as $R(p, x)=p \int_{0}^{x} q(p, v) d v$; hence,

$$
\begin{aligned}
R_{p}(p, x) & =\int_{0}^{x}\left(q(p, v)+p q_{p}(p, v)\right) d v \\
& =\int_{0}^{x} q(p, v)(1-\mathscr{E}(p, v)) d v,
\end{aligned}
$$

where the second equality is obtained using (5).

Lemma 2. (a) $p^{*}(x)$ decreasing in $x \Leftrightarrow \mathscr{E}^{*}(x) \geqslant 1$. (b) $x^{*}(p)$ decreasing in $p \Leftrightarrow \mathscr{E}^{*}(p) \geqslant 1$.

Proof. (a) Optimality of $p^{*}(x)$ and the implicit function theorem imply $\partial p^{*}(x) / \partial x=-\Pi_{x p}^{*}(x) / \Pi_{p p}^{*}(x)$. The denominator is negative by the second-order condition. It remains to show that the numerator is negative. Indeed, (6) and the assumption of the lemma imply $\Pi_{x p}^{*}(x)=q^{*}(x)(1-$ $\left.\mathscr{E}^{*}(x)\right) \leqslant 0$. Part (b) is analogous.

Lemma 3. For all $p \geqslant p_{L}, p_{L} q\left(p_{L}, x^{*}(p)\right) \geqslant c$. Moreover, $\mathscr{E}^{*}\left(p_{L}\right)=1$ or $p_{L}=c$.

Table 3. Summary of notation.

\begin{tabular}{ll}
\hline$c$ & Unit cost \\
$x$ & Inventory, stock, or order quantity \\
$p$ & Price \\
$p_{L}$ & Lower bound on price, $p \geqslant p_{L}$ \\
$p^{*}(x)$ & Optimal price for a given quantity $x$ \\
$x^{*}(p)$ & Optimal quantity for a given price $p$ \\
$p^{* *}, x^{* *}$ & Optimal NVP price and quantity \\
$\mathbf{D}(p)=d(p, \mathbf{Z})$ & Stochastic price-dependent demand \\
$\mathbf{Z}$ & Random component of $\mathbf{D}(p)$ \\
$d(p, z)$ & Deterministic (riskless) demand function \\
$z(p, x)$ & Stocking factor, $d(p, z(p, x))=x$ \\
$r(p, x)=p d(p, z)$ & Riskless revenue function \\
$R(p, x)$ & Expected revenue function \\
$\Pi(p, x)=R(p, x)-c x$ & Expected profit function \\
$\phi(z) ; \Phi(z)=P(\mathbf{Z} \leqslant z)$ & Density; resp., cdf of $\mathbf{Z}$ \\
$f(p, x) ; F(p, x)=P(\mathbf{D}(p) \leqslant x)$ & Density; resp., cdf of $\mathbf{D}(p)$ \\
$q(p, x)=1-F(p, x)$ & Lost-sales rate; survival function of $\mathbf{D}(p)$ \\
$\mathscr{E}(p, x)$ & Elasticity of the lost-sales rate $q(p, x)$ \\
$h_{D}(p, x), g_{D}(p, x)$ & Hazard (or failure) rate, resp., generalized hazard rate of $\mathbf{D}(p)$ \\
$h_{Z}(z), g_{Z}(z)$ & Hazard rate, resp., generalized hazard rate of $\mathbf{Z}$ \\
$\epsilon_{P}(p, x)=\frac{-p d_{p}(p, z(p, x))}{d(p, z(p, x))}$ & Riskless price elasticity \\
\hline
\end{tabular}


Proof. By definition, $p_{L}=\arg \max \left\{d\left(p, \bar{\Phi}^{-1}(c / p)\right)\right.$; $p \geqslant c\}=\arg \max \left\{d\left(p, z^{*}(p)\right) ; p \geqslant c\right\}=\arg \max \left\{x^{*}(p)\right.$; $p \geqslant c\}$. Hence, $x^{*}(p) \leqslant x^{*}\left(p_{L}\right)$ for all $p \geqslant p_{L}$. It follows that for all $p \geqslant p_{L}, q\left(p_{L}, x^{*}(p)\right) \geqslant q\left(p_{L}, x^{*}\left(p_{L}\right)\right)=c / p_{L}$. For the second part, if $p_{L}>c$, then $p_{L}$ is an interior maximizer of $x^{*}(p)$. Therefore, by the implicit function theorem, $\Pi_{x p}^{*}\left(p_{L}\right)=0$, which amounts to $\mathscr{E}^{*}\left(p_{L}\right)=1$.

Proof of Theorem 1. (a) The first part follows by Lemma 2. It remains to show that $\mathscr{E}(p, x)$ increasing in $x$ implies $\mathscr{E}^{*}(x) \geqslant 1$. Using Lemma $1(\mathrm{~b})$, write

$$
\begin{aligned}
R_{p}(p, x)=\int_{0}^{x} Q(p, v) d v, & \\
& \text { where } Q(p, x)=q(p, x)(1-\mathscr{E}(p, x)) .
\end{aligned}
$$

Because $\mathscr{E}(p, x)$ is increasing in $x$ and $q(p, x) \geqslant 0$, it follows that for any fixed $p, Q(p, x)$ crosses zero at most once, and from above. Therefore, if $Q^{*}(x)=Q\left(p^{*}(x), x\right)>$ 0 , it follows that for any $v \leqslant x, Q\left(p^{*}(x), v\right)>0$; hence, $\int_{0}^{x} Q\left(p^{*}(x), v\right) d v>0$, contradicting the first-order condition, $R_{p}(p, x)=\int_{0}^{x} Q\left(p^{*}(x), v\right) d v=0$. It follows that $Q^{*}(x)=Q\left(p^{*}(x), x\right) \leqslant 0$, that is, $\mathscr{E}^{*}(x) \geqslant 1$.

(b) The first part follows by Lemma 2 . It remains to show that $\mathscr{E}(p, x)$ increasing in $p$ implies $\mathscr{E}^{*}(p) \geqslant 1$. The optimality condition for $x$ (Lemma 1a) states that the following expression, evaluated at $x^{*}(p)$, equals zero:

$$
\begin{aligned}
p q(p, x)-c & =\int_{p_{L}}^{p} \frac{\partial}{\partial v}(v q(v, x)-c) d v+p_{L} q\left(p_{L}, x\right)-c \\
& =\int_{p_{L}}^{p} q(v, x)(1-\mathscr{E}(v, x)) d v+\left(p_{L} q\left(p_{L}, x\right)-c\right) .
\end{aligned}
$$

By the first part of Lemma 3, the second term, evaluated at $x^{*}(p)$, is nonnegative. Therefore, the first term must be nonpositive at $x^{*}(p)$, i.e., $\int_{p_{L}}^{p} Q\left(v, x^{*}(p)\right) d v \leqslant 0$, where $Q(p, x)=q(p, x)(1-\mathscr{E}(p, x))$. Because $\mathscr{E}(p, x)$ increases in $p$, and $q(p, x) \geqslant 0, Q(p, x)$ crosses zero at most once, and from above, as $p$ increases. Therefore, $Q\left(p, x^{*}(p)\right) \leqslant$ 0 , i.e., $\mathscr{E} *(p) \geqslant 1$.

Proof of Proposition 2. We show that the Hessian matrix of $\Pi(p, x)$ is negative semidefinite. The secondorder derivatives are

$$
\begin{aligned}
& \Pi_{x x}(p, x)=-p f(p, x), \\
& \Pi_{x p}(p, x)=q(p, x)+p f(p, x) d_{p}(p, z), \\
& \Pi_{p p}(p, x)=\mathbb{E}\left[r_{p p}(p, \mathbf{Z}) ; \mathbf{\Omega}\right]-p f(p, x) d_{p}^{2}(p, z(p, x)),
\end{aligned}
$$

where $\boldsymbol{\Omega}=\boldsymbol{\Omega}(p, x)=(\mathbf{D}(p) \leqslant x)$ defines the event of no lost sales (arguments are omitted for notational convenience), and we use the standard notation $\mathbb{E}[\mathbf{A} ; \mathbf{B}]=$ $\mathbb{E}[\mathbf{A} \mid \mathbf{B}] P(\mathbf{B})$. Equation (11) is obtained by differentiating twice with respect to $p$ the objective in (1), written as

$$
\Pi(p, x)=p x q(p, x)+\mathbb{E}[r(p, \mathbf{Z}) ; \mathbf{\Omega}]-c x .
$$

Because $\Pi_{x x} \leqslant 0$ and $\Pi_{p p} \leqslant 0$ (from concavity of $r$ ), it remains to check that the determinant of the Hessian, $\Delta(p, x)$, is positive. Pairing up terms, we obtain

$$
\begin{aligned}
\Delta(p, x)= & \Pi_{x x}(p, x) \Pi_{p p}(p, x)-\Pi_{x p}^{2}(p, x) \\
= & -p f(p, x) \mathbb{E}\left[r_{p p}(p, \mathbf{Z}) ; \mathbf{\Omega}\right]-q(p, x) \\
& \cdot\left[q(p, x)+2 p f(p, x) d_{p}(p, z(p, x))\right] \\
= & -p f(p, x) \mathbb{E}\left[r_{p p}(p, \mathbf{Z}) ; \mathbf{\Omega}\right] \\
& +q(p, x)^{2}(2 \mathscr{E}(p, x)-1) .
\end{aligned}
$$

The last equality follows from an equivalent expression for LSR elasticity:

$\mathscr{E}(p, x)=\frac{-p f(p, x) d_{p}(p, z(p, x))}{q(p, x)}$,

because $F_{p}(p, x)=\partial / \partial p P(\mathbf{D}(p) \geqslant x)=f(p, x)$. $d_{p}(p, z(p, x))$. The first term in (15) is positive by concavity of $r$, and the second because $\mathscr{E} \geqslant 1 / 2$.

Proof of Theorem 2. By the envelope theorem, we have

$$
\frac{\partial^{2}}{\partial x^{2}} \Pi^{*}(x)=\Pi_{x x}(p, x)-\left.\frac{\Pi_{x p}^{2}(p, x)}{\Pi_{p p}(p, x)}\right|_{p=p^{*}(x)} .
$$

From the second-order condition, $\Pi_{p p}^{*}(x)<0$. Using (15), we write

$$
\begin{aligned}
\Delta^{*}(x) & =\Pi_{x x}^{*}(x) \Pi_{p p}^{*}(x)-\Pi_{x p}^{*}(x)^{2} \\
& =-p f^{*}(x) \mathbb{E}\left[r_{p p}^{*} ; \boldsymbol{\Omega}^{*}\right]-q^{*}(x)^{2}\left(2 \mathscr{E}^{*}(x)-1\right),
\end{aligned}
$$

which is positive whenever $\mathscr{E}^{*}(x) \geqslant 1 / 2$ and $r_{p p} \leqslant 0$. It follows that $\partial^{2} / \partial x^{2} \Pi^{*}(x) \leqslant 0$ and $\Pi^{*}(x)$ is concave in $x$. Part (b) is proved analogously. By Theorem $1, \mathscr{E}(p, x)$ increasing in $p$ or $x$ guarantees the LSR elasticity bound required by parts (a) and (b) of the proposition, which settles part (c).

Proof of ReMARK 1. The proof follows from $r_{p p}=0$ and the proof of Proposition 2 and Theorem 2.

Proof of Proposition 3. Because

$$
\mathscr{E}(p, x)=p \frac{-q_{p}(p, x)}{q(p, x)}=-p \frac{\partial}{\partial p} \log q(p, x),
$$

we obtain

$$
\begin{aligned}
\frac{\partial}{\partial x} \frac{\mathscr{E}(p, x)}{p} & =-\frac{\partial}{\partial p} \frac{\partial}{\partial x} \log q(p, x) \\
& =\frac{\partial}{\partial p} \frac{f(p, x)}{q(p, x)}=\frac{\partial}{\partial p} h_{D}(p, x) .
\end{aligned}
$$

Therefore, $\mathscr{E}$ increasing in $x$ is equivalent to $h_{D}$ increasing in $p$. For the second part, letting $g_{D}(p, x)=x h_{D}(p, x)$ denote the GFR of $\mathbf{D}(p)$, observe that

$$
\mathscr{E}(p, x)=\frac{-p f(p, x) d_{p}(p, z(p, x))}{q(p, x)}=g_{D}(p, x) \epsilon_{P}(p, x),
$$

where the first equality follows from (16) and the second by definition of $g_{D}$ and $\epsilon_{P}$. 
The proof of Proposition 4 relies on the following lemma:

LEMMA 4. (a) $\mathscr{E}(p, x)$ is increasing in $x$ if and only if $\tilde{\mathscr{E}}(p, z)=\mathscr{E}(p, d(p, z))$ is increasing in $z$.

(b) $\mathscr{E}(p, x)$ is increasing in $p$ if $\mathscr{E}(p, z)$ is increasing in both $z$ and $p$.

Proof. Differentiating $\tilde{\mathscr{E}}(p, z(p, x))=\mathscr{E}(p, x)$ on both sides with respect to $x$ (respectively, $p$ ) we have $\mathscr{E}_{x}(p, x)=$ $\tilde{\mathscr{E}}_{z}(p, z(p, x)) z_{x}(p, x)$ (respectively, $\tilde{\mathscr{E}}_{z}(p, z(p, x)) z_{p}(p, x)$ $\left.+\tilde{\mathscr{E}}_{p}(p, z(p, x))=\mathscr{E}_{p}(p, x)\right)$. The result follows because $z_{x}(p, x)=1 /\left(d_{z}(p, z(p, x))\right) \geqslant 0$, and $z_{p}(p, x)=$ $\left(-d_{p}(p, z(p, x))\right) /\left(d_{z}(p, z(p, x))\right) \geqslant 0$.

Proof of Proposition 4. (a) Writing $\tilde{\mathscr{E}}(p, z)=$ $h_{Z}(z)\left(-p d_{p}(p, z)\right) /\left(d_{z}(p, z)\right)$, we have

$\frac{\partial \tilde{\mathscr{E}}(p, z)}{\partial z}=-p h_{Z}^{\prime}(z) \frac{d_{p}}{d_{z}}-p h_{Z}(z) \frac{\partial}{\partial z} \frac{d_{p}}{d_{z}}$.

This and Lemma $4($ a) imply $\mathscr{E}(p, x)$ is increasing in $x$ when $h_{Z}(z)$ is increasing and $d_{p} / d_{z}$ is decreasing in $z$. The second part follows from $\tilde{\mathscr{E}}_{p}(p, z)=h_{Z}(z)(\partial / \partial p)\left(p d_{p} / d_{z}\right)$ via Lemma $4(\mathrm{~b})$ because $p d_{p} / d_{z}$ is decreasing in $p$. Part (b) follows because $\tilde{\mathscr{E}}(p, z)=g_{Z}(z) \tilde{\epsilon}_{P Z}(p, z)$ and from Lemma 4 (a) by writing

$\frac{\partial \tilde{\mathscr{E}}(p, z)}{\partial z}=p g_{Z}^{\prime}(z) \tilde{\epsilon}_{P Z}(p, z)+p g_{Z}(z) \frac{\partial \tilde{\epsilon}_{P Z}(p, z)}{\partial z}$.

Proof of Corollary 2. We focus on $\mathscr{E}(p, x)$ increasing in $x$, which, by Proposition 3 , is equivalent to $h_{D}(p, x)$ is increasing in $p$. Throughout the proof we use the general fact that $z_{p}(p, x)=\left(-d_{p}(p, z(p, x))\right) /$ $\left(d_{z}(p, z(p, x))\right) \geqslant 0$. For additive-multiplicative models, $z(p, x)=(x-\beta(p)) /(\alpha(p))$, which allows us to write

$h_{D}(p, x)=\frac{h_{Z}(z(p, x))}{d_{z}(z(p, x))}=\frac{h_{Z}(z(p, x))}{\alpha(p)}=\frac{g_{Z}(z(p, x))}{x-\beta(p)}$.

(a) If $\mathbf{Z}$ is IFR because $z_{p} \geqslant 0$, and $\alpha$ is decreasing, we obtain

$\frac{\partial}{\partial p} h_{D}(p, x)=h_{Z}^{\prime}(z(p, x)) \frac{z_{p}(p, x)}{\alpha(p)}-h_{Z}(z(p, x)) \frac{\alpha^{\prime}(p)}{\alpha^{2}(p)} \geqslant 0$.

On the other hand, because $z_{p} \geqslant 0$ and $\beta$ is decreasing, if

$$
\begin{aligned}
\frac{\partial}{\partial p} h_{D}(p, x)= & \frac{\partial}{\partial p} \frac{g_{Z}(z(p, x))}{x-\beta(p)} \\
= & g_{Z}^{\prime}(z(p, x)) \frac{z_{p}(p, x)}{x-\beta(p)} \\
& +g_{Z}(z(p, x)) \frac{\beta^{\prime}(p)}{(x-\beta(p))^{2}} \geqslant 0,
\end{aligned}
$$

then $g_{Z}$ must be increasing, i.e., $\mathbf{Z}$ is IGFR. (b) For the multiplicative case, $\beta(p)=0$, so

$h_{D}(p, x)=\frac{1}{\alpha(p)} h_{Z}\left(\frac{x}{\alpha(p)}\right)=\frac{1}{x} g_{Z}(z(p, x))$.

Again, because $z_{p} \geqslant 0$, we have that $h_{D}$ is increasing in $p$ if and only if $g_{Z}$ is increasing, i.e., $\mathbf{Z}$ is IGFR.

(c) For the additive case, $\alpha(p)=1$, so $\partial / \partial p$. $\left(h_{D}(p, x)\right)=h_{Z}^{\prime}(z(p, x)) z_{p}(p, x)$. Because $z_{p} \geqslant 0, h_{D}$ is increasing in $p$ if and only if $h_{Z}$ is increasing, i.e., $\mathbf{Z}$ is IFR.

The conditions for $\mathscr{E}(p, x)$ increasing in $p$ follow directly from Corollary 1 for parts (a) and (c), whereas part (b) follows from Proposition 4(b).

Proof of the Results in Table 2. For additive-linear models, $z(p, x)=x+b p$, and $\mathscr{E}(p, x)=b p h_{Z}(b p+x)=$ $b p /(b p+x) g_{Z}(x+b p)$. The first expression shows that this is increasing in $x$ iff $h_{Z}$ is increasing. The last expression indicates that $g_{Z}$ increasing implies $\mathscr{E}$ increasing in $p$, because $b p /(b p+x)$ is increasing in $p$. Setting $x=0$, we see that IGFR is also necessary for $\mathscr{E}$ increasing in $p$.

For the multiplicative isoelastic model, $z(p, x)=x p^{b} / a$ and $\mathscr{E}(p, x)=b g_{Z}\left(x p^{b} / a\right)$. This is increasing in $x$ (respectively, $p$ ) if and only if $g_{Z}$ is increasing.

For the power model, $z(p, x)=x p^{b} /(a-x)$ and $\mathscr{E}(p, x)=b g_{Z}\left(x p^{b} / a-x\right)$. This is increasing in $x$ (respectively, $p$ ) if and only if $g_{Z}$ is increasing (because its argument is increasing in both $x$ and $p$ ).

For the logit model, $z(p, x)=b p+\ln x /(a-x), x \leqslant a$, and $\mathscr{E}(p, x)=b p h_{Z}(b p+\ln x /(a-x))=b p(b p+\ln x /$ $\left.(a-x) g_{Z}(x+b p)\right)$, where $\ln =\log _{e}$. The first expression shows that this is increasing in $x$ iff $h_{Z}$ is increasing, because the argument of $h_{Z}$ is increasing in $x$. The second indicates that $g_{Z}$ increasing implies $\mathscr{E}$ increasing in $p$, because the fraction preceding $g_{Z}$ is increasing in $p$. Setting $x=a / 2$, we see that IGFR is also necessary for $\mathscr{E}$ increasing in $p$.

For the exponential, $z(p, x)=b p+\ln x$ and $\mathscr{E}(p, x)=$ $b h_{Z}(b p+\ln x)$. This is increasing in $x$ (respectively, $p$ ) iff $h_{Z}$ is increasing.

For the base- $a$ log model, $z(p, x)=b p+a^{x}$ and $\mathscr{E}(p, x)=b p h_{Z}\left(b p+a^{x}\right)=b p /\left(b p+a^{x}\right) g_{Z}\left(b p+a^{x}\right)$. The first expression shows that this is increasing in $x$ iff $h_{Z}$ is increasing. The second indicates that $g_{Z}$ increasing implies $\mathscr{E}$ increasing in $p$, because the fraction in front of $g_{Z}$ is increasing in $p$.

For the economic wtp model, the result follows from Corollary 2(b); the elasticity of the riskless demand $\alpha(p)=$ $P(\mathbf{W} \geqslant p)$ is precisely the GFR of the willingness-to-pay distribution $\mathbf{W}$.

The proof of Proposition 5 relies on the following additional lemma.

Lemma 5. (a) If $\pi_{p z} \leqslant 0$, the optimal base price $p_{B}(z)$ is decreasing in $z$.

(b) If $\pi_{p z} \geqslant 0$, the optimal base price $p_{B}(z)$ is increasing in $z$. If , moreover, $d_{p z} \leqslant 0$, then the optimal price $p^{*}(z)$ is increasing in $z$. 
Proof. For $p_{B}(z)$, by definition, $p_{B}(z)$ optimizes $\widetilde{\Pi}^{B}(p, z)$ $=(p-c) \mathbb{E}[d(p, \min (\mathbf{Z}, z))]=\mathbb{E}[\pi(p, \min (\mathbf{Z}, z))]$. Therefore, $\widetilde{\Pi}_{p z}^{B}(p, z)=\pi_{p z}(p, z) \bar{\Phi}(z)$, and the sign of $\pi_{p z}$ dictates monotonicity of $p_{B}(z)$, by Topkis' Theorem.

For $p^{*}(z)$, letting $\widetilde{\Pi}(p, z)=\Pi(p, d(p, z))=\mathbb{E}[r(p$, $\min (\mathbf{Z}, z)]-c d(p, z)$, we have

$$
\begin{aligned}
\widetilde{\Pi}_{p z}(p, z) & =r_{p z}(p, z) \bar{\Phi}(z)-c d_{p z}(p, z) \\
& =\pi_{p z}(p, z) \bar{\Phi}(z)-c d_{p z}(p, z) \Phi(z) \geqslant 0
\end{aligned}
$$

when $\pi_{p z} \geqslant 0$ and $d_{p z} \leqslant 0$. Therefore, $p^{*}(z)$ is increasing in $z$ by Topkis' Theorem.

Proof of Proposition 5. For part (a), by definition, $p_{B}(z)$ solves $\mathbb{E}\left[\pi_{p}(p, \min (\mathbf{Z}, z))\right]=0$, so

$$
\left.\widetilde{\Pi}_{p}(p, z)\right|_{p=p_{B}(z)}=c \mathbb{E}\left[d_{p}\left(p_{B}, \min (\mathbf{Z}, z)\right)-d_{p}\left(p_{B}, z\right)\right] \leqslant 0,
$$

because $d_{p}(p, z)$ is increasing in $z$. It follows that $p^{*}(z) \leqslant$ $p_{B}(z)$. For the second part, we write $p_{B}(\infty)=\arg \max _{p}(p-$ c) $\mathbb{E}[d(p, \min (\mathbf{Z}, \infty))]=\arg \max _{p}(p-c) \mathbb{E}[\mathbf{D}(p)]=p^{0}$, by abuse of notation. Therefore, for the optimal $z^{* *}=$ $\arg \max \widetilde{\Pi}\left(p^{*}(z), z\right)$, we have

$p^{0}=p_{B}(\infty) \geqslant p_{B}\left(z^{* *}\right) \geqslant p^{*}\left(z^{* *}\right)=p^{* *}$,

where the first inequality follows from Lemma 5(b) $\left(d_{p z} \geqslant 0\right.$ implies $\pi_{p z}=(p-c) d_{p z}+d_{z} \geqslant 0$ because $\left.d_{z} \geqslant 0\right)$, and the second inequality from the first part of the proposition.

The proof of part (b) is analogous to (a), yielding the opposite inequalities than in (18), based on Lemma 5(a), which relies on submodularity of $\pi$. The result for multiplicative demand follows directly from the firstorder conditions. Indeed, $p^{*}(z)$ solves $\pi^{\prime}(p) \mathbb{E}[\min (\mathbf{Z}, z)]-$ $c \alpha^{\prime}(p) \mathbb{E}[z-\mathbf{Z}]^{+}=0$. The left-hand side evaluated at $p^{0}$ is positive because $\alpha$ is decreasing, and $\pi^{\prime}\left(p^{0}\right)=0$ (by optimality of $\left.p^{0}\right)$ so $p^{0} \leqslant p^{*}(z)$, in particular $p^{0} \leqslant p^{* *}$.

Proof of Proposition 6. From Topkis' Theorem, for the first part, it is sufficient to show that $\mathbb{E}\left[r\left(p_{2}\right.\right.$, $\left.\left.\min \left(\mathbf{Z}_{L}, z\right)\right)-r\left(p_{1}, \min \left(\mathbf{Z}_{L}, z\right)\right)\right] \geqslant \mathbb{E}\left[r\left(p_{2}, \min (\mathbf{Z}, z)\right)-\right.$ $\left.r\left(p_{1}, \min (\mathbf{Z}, z)\right)\right]$ for $p_{2} \geqslant p_{1}$. If $r_{p}$ is increasing in $z$, then for arbitrarily fixed $z, u(y)=r\left(p_{2}, \min (y, z)\right)-$ $r\left(p_{1}, \min (y, z)\right)$ is increasing in $y$. Because $\mathbf{Z}_{L} \succeq_{F S D} \mathbf{Z}$, it follows that $\mathbb{E}\left[u\left(\mathbf{Z}_{L}\right)\right] \geqslant \mathbb{E}[u(\mathbf{Z})]$, which completes the proof. For the second part, if $r_{p}$ is increasing and concave in $z$, then for arbitrarily fixed $z, u(y)=r\left(p_{2}, \min (y, z)\right)-$ $r\left(p_{1}, \min (y, z)\right)$ is concave in $y$. Because $\mathbf{Z}_{V} \succeq_{C X} \mathbf{Z}$, it follows that $\mathbb{E}\left[u\left(\mathbf{Z}_{V}\right)\right] \leqslant \mathbb{E}[u(\mathbf{Z})]$. Because $\mathbf{Z} \succeq_{C X} \mu$, these results enable the comparison of $p^{*}(z)$ and $p^{0}(z)$. Part (b) is proved analogously.

\section{Endnotes}

1. Our results also extend existing cost sensitivity results (e.g., Ha 2001, Yao et al. 2006). It is well known that $x^{* *}(c)$ decreases in $c$, but the optimal price is not necessarily monotone. In fact, $p^{* *}(c)$ is increasing in $c$ if and only if $p^{*}(x)$ increases with $x$, so in particular if $\mathscr{E}(p, x)$ is increasing in $x$, by Theorem 1 .

2. By definition, $\mathbf{X}$ is smaller than $\mathbf{Y}$ in the hazard-rate order $\left(\mathbf{Y} \succeq_{F R} \mathbf{X}\right)$ if and only if their respective hazard rates satisfy $h_{\mathbf{X}}(z) \geqslant h_{\mathbf{Y}}(z)$ (Müller and Stoyan 2002, Theorem 1.3.3). The hazard-rate order is theoretically stronger than first-order dominance and weaker than likelihood-ratio order; however, the three are actually equivalent for most parametric families of distributions with the natural parameter order (see Müller and Stoyan 2002, Table 1.1).

3. By definition, (1) $\mathbf{X}$ first-order dominates $\mathbf{Y}\left(\mathbf{X} \succeq_{F S D} \mathbf{Y}\right)$ if $\mathbb{E}[u(\mathbf{X})] \geqslant \mathbb{E}[u(\mathbf{Y})]$ for all increasing functions $u$, or equivalently, if $P(\mathbf{X}>t) \geqslant P(\mathbf{Y}>t)$ for all $t$; (2) $\mathbf{X}$ dominates $\mathbf{Y}$ in the convex order $\left(\mathbf{X} \succeq_{C X} \mathbf{Y}\right)$ if $\mathbb{E}[u(\mathbf{X})] \geqslant$ $\mathbb{E}[u(\mathbf{Y})]$ for all convex functions $u$. If $\mathbf{X} \succeq_{C X} \mathbf{Y}$, then $\mathbb{E}[\mathbf{X}]=\mathbb{E}[\mathbf{Y}]$, and $\operatorname{Var}(\mathbf{X}) \geqslant \operatorname{Var}(\mathbf{Y})$ (see Müller and Stoyan 2002).

\section{Acknowledgments}

The authors thank the review team for valuable suggestions that improved this manuscript. They also thank James Dana, Ye Lu, Guillaume Roels, Garrett van Ryzin, and Kalyan Talluri for insightful comments on various versions of the paper.

\section{References}

Agrawal, V., M. Ferguson. 2007. Bid-response models for customised pricing. J. Pricing Revenue Management 6(3) 212-228.

Agrawal, V., S. Seshadri. 2000. Impact of uncertainty and risk aversion on price and order quantity in the newsvendor problem. Manufacturing Service Oper. Management 2 410-423.

Bagnoli, M., T. Bergstrom. 2005. Logconcave probability and its applications. Econom. Theory 26(2) 445-469.

Barlow, R. E., F. Proschan. 1996. Mathematical Theory of Reliability. G. H. Golub, ed. SIAM Classics in Applied Mathematics. Society for Industrial and Applied Mathematics, Philadelphia.

Bernstein, F., A. Federgruen. 2004. A general equilibrium model for industries with price and service competition. Oper. Res. 52(6) 868-886.

Bernstein, F., A. Federgruen. 2005. Decentralized supply chains with competing retailers under demand uncertainty. Management Sci. 51(1) 18-29.

Chan, L. M. A., Z. J. Shen, D. Simchi-Levi, J. L. Swann. 2004. Coordination of pricing and inventory decisions: A survey and classification D. Simchi-Levi, S. D. Wu, Z. J. Shen, eds. Handbook of Quantitative Supply Chain Analysis: Modeling in the E-Business Era. Kluwer Academic Publishers, Dordrecht, The Netherlands, 335-392.

Chen, X., D. Simchi-Levi. 2003. Coordinating inventory control and pricing strategies with random demand and fixed ordering cost: The finite horizon case. Oper. Res. 52(6) 887-896.

de Vericourt, F., M. S. Lobo. 2009. Resource and revenue management in nonprofit operations. Oper. Res. 57(5) 1114-1128.

Federgruen, A., A. Heching. 1999. Combined pricing and inventory control under uncertainty. Oper. Res. 47(3) 454-475. 
Ha, A. 2001. Supplier-buyer contracting: Asymmetric cost information and cutoff level policy for buyer participation. Naval Res. Logist. 48(1) 41-64.

Hanssens, D., L. Parsons, R. Schultz. 2001. Market Response Models, Econometric and Times Series Analysis. Kluwer Academic Publishers, Dordrecht, The Netherlands.

Karlin, S., C. R. Carr. 1962. Prices and Optimal Inventory Policy in Studies in Applied Probability and Management Science. Stanford University Press, Stanford, CA.

Kocabıyıkoğlu, A., I. Popescu, C. Stefanescu. 2009. Pricing and revenue management: Coordinated vs. hierarchical approaches. Working paper, INSEAD, Singapore.

Lariviere, M. 2006. A note on probability distributions with increasing generalized failure rates. Oper. Res. 54(3) 602-604.

Lariviere, M., E. L. Porteus. 2001. Selling to the newsvendor: An analysis of price-only contracts. Manufacturing Service Oper. Management 3(4) 293-305.

Lau, H., S. Lau. 1988. The newsvendor problem with price dependent demand distribution. IIE Trans. 20(2) 168-175.

Li, Q., D. Atkins. 2005. On the effect of demand randomness on a price/quantity setting firm. IIE Trans. 37(12) 1143-1153.

Mills, E. S. 1959. Uncertainty and price theory. Quart. J. Econom. 73(1) 116-130.

Mills, E. S. 1962. Price, Output and Inventory Policy. John Wiley \& Sons, New York.

Monahan, G. E., N. C. Petruzzi, W. Zhao. 2004. The dynamic pricing problem from a newsvendor's perspective. Manufacturing Service Oper. Management 6(1) 73-91.

Müller, A., D. Stoyan. 2002. Comparison Methods for Stochastic Models and Risks. John Wiley \& Sons, New York.

Netessine, S. 2006. Dynamic pricing of inventory/capacity with infrequent price changes. Eur. J. Oper. Res. 174(1) 553-580.

Netessine, S., N. Rudi. 2003. Centralized and competitive inventory models with demand substitution. Oper. Res. 51(2) 329-335.

Nevins, A. J. 1966. Some effects of uncertainty: Simulation of a model of price. Quart. J. Econom. 80 (1) 73-87.
Petruzzi, N. C., M. Dada. 1999. Pricing and newsvendor problem: A review with extensions. Oper. Res. 47(2) 183-194.

Phillips, R. L. 2005. Pricing and Revenue Optimization. Stanford Business Books, Stanford, CA.

Raz, G., E. Porteus. 2006. A fractiles perspective to the joint price/quantity newsvendor model. Management Sci. 52(11) 1764-1777.

Tayur, S., R. Gareshan, M. Magazine. 1999. Quantitative Models for Supply Chain Management. Kluwer Academic Publishers, Dordrecht, The Netherlands.

Topkis, D. M. 1998. Supermodularity and Complementarity. Princeton University Press, Princeton, NJ.

Wang, Y., L. Jiang, Z.-J. Shen. 2004. Channel performance under consignment contract with revenue sharing. Management Sci. 50(1) 34-47.

Whitin, T. M. 1955. Inventory control and price theory. Management Sci. 2(1) 61-68.

Yano, C., S. M. Gilbert. 2003. Coordinated pricing and production/procurement decisions: A review. J. Eliashberg, A. Chakravarty, eds. Managing Business Interfaces: Marketing, Engineering, and Manufacturing Perspectives. Kluwer, Norwell, MA.

Yao, L., Y. F. Chen, H. Yan. 2006. The newsvendor problem with pricing. Internat. J. Management Sci. Engrg. Management 1(1) 3-16.

Young, L. 1978. Price, inventory, and the structure of uncertain demand. New Zealand Oper. Res. J. 6(2) 157-177.

Zabel, E. 1970. Monopoly and uncertainty. Rev. Econom. Stud. 37(2) 205-219.

Zabel, E. 1972. Multiperiod monopoly and uncertainty. J. Econom. Theory 5 524-536.

Zhao, X., D. R. Atkins. 2008. Newsvendors under simultaneous price and inventory competition. Manufacturing Service Oper. Manage ment 10(3) 539-546.

Ziya, S., H. Ayhan, D. Foley. 2004. Relationship among three assumptions in revenue management. Oper. Res. 52(5) 804-809.

Ziya, S., H. Ayhan, D. Foley. 2006. Optimal prices for finite capacity queueing systems. Oper. Res. Lett. 34 214-218. 